\title{
MODULAR FORMS AND TOPOLOGY
}

\author{
KEFENG LIU
}

We want to discuss various applications of modular forms in topology. The starting point is elliptic genus and its generalizations. The main techniques are the Atiyah-Singer index theorem, the Atiyah-Bott-Segal-Singer Lefschetz fixed point formula, Kac-Moody Lie algebras, modular forms and theta-functions. Just as the representations theory of classical Lie groups has close connections with the Atiyah-Singer index formula as exposed in [A1], the representation theory of loop groups plays very important role in our study. One of the most important new features of loop group representations is the modular invariance of the Kac-Weyl character formula, which allows us to derive many interesting new results and to unify many important old results in topology. In this paper we will develope along this line. We hope that the other features of loop group representations, such as fusion rules and tensor category structure may also be applied to topology. See the discussions in $\S 3$.

The contents of this paper is organized in the following way. In $\S 1$ we introduce elliptic genus by combining index theory and the representation theory of loop groups. The relation of the classical index theory with representation theory of classical Lie groups was discussed in [A1]. Here one finds that just replacing the classical Lie groups by their corresponding loop groups, we get the complete theory of elliptic genus, or more generally the index theory on loop space. Especially the Dirac operator and the Witten genus of loop space are derived more convincingly in this way. Then all of the other well-known properties of elliptic genus, such as functional equations, characterizations by rigidity and fibrations, can be easily obtained. Here we only pick some less well-known results to discuss, for example the expressions of the parameters in elliptic genera in terms of theta-functions.

Different from the classical Lie group case, a new feature in our situation appears, the modular invariance which, by combining with index theory, is applied to obtain many new topological results. This is the content of $\S 2$. Most results in this section are special cases of more general results. For simplicity we only give the main ideas of the proofs.

We make some geometric constructions in $\S 3$ to understand elliptic cohomology. The construction in $\S 3.3$ is motivated by the vertex operator algebra construction of the monstrous moonshine module. In $\S 3.1$ we introduce vector

1991 Mathematics Subject Classification. 55P35, 55N91, 58G10. 
bundles with infinite dimensional structure groups. We study the corresponding Grothendieck groups and the Riemann-Roch properties in $\S 3.2$. An easy corollary is that elliptic genus can be realized as the difference of two infinite dimensional vector bundles on a sphere with the action of Virasoro algebra on each of them. For each modular subgroup, a ring of graded bundles with modularity is introduced in $\S 3.3$, to which there is a natural homomorphism from elliptic cohomology. Several simple theorems are stated without proof. The detail of this section will appear in a forthcoming paper.

This survey article is basically an expanded version of my lectures given in the topology seminars at MIT, Harvard, and the AMS conference on the monster and the moonshine module. Many ideas are certainly already wellknown to experts. The reader may find related discussions in the references. I have also benefited from discussions with many people. I would like to thank the organizers and the audience of the seminars and conference, especially $\mathrm{R}$. Bott, J.-L. Brylinski, C. Dong, M. Hopkins, Y. Huang, V. Kac, G. Katz, H. Miller, A. Radul, W. Wang, E. Weinstein, S.-T. Yau, Y. Zhu.

\section{Contents}

1. Index theory, elliptic curves and loop groups 2

1.1. Atiyah-Singer Index theorem 2

1.2. Loop groups and index theory 4

1.3. Elliptic genera 6

1.4. Elliptic genera and theta-functions 8

2. Applications in topology 10

2.1. Miraculous cancellation formula 10

\begin{tabular}{ll}
2.2. & 13 \\
\hline
\end{tabular}

2.3. A vanishing theorem of the Witten genus 15

$\begin{array}{ll}\text { 2.4. Kac-Moody algebras in topolgy } & 17\end{array}$

3. Vector bundles and modular forms 18

3.1. Infinite dimensional vector bundles 18

3.2. Riemann-Roch and Virasoro algebra $\quad 19$

3.3. Vector bundles and Jacobi forms 20

3.4. Relation with elliptic cohomology 22

$\begin{array}{ll}\text { References } & 24\end{array}$

\section{INDEX THEORY, ELLIPTIC CURVES AND LOOP GROUPS}

One can look at elliptic genus from several different points of view; from index theory, from representation theory of Kac-Moody affine Lie algebras or from the theory of elliptic functions and modular forms. Each of them shows 
us some quite different interesting features of ellitic genus. On the other hand we can also combine the forces of these three different mathematical fields to derive many interesting results in topology such as rigidity, divisibility and vanishing of topological invariants.

In this section, we first introduce elliptic genus by combing the representation theory of affine Lie algebras and the Atiyah-Singer index theory from which we derive all the other properties of elliptic genus such as functional equations and logrithms, etc. This section is a 'loop' analogue of [A1].

1.1. Atiyah-Singer Index theorem. Let $M$ be a smooth compact spin manifold of dimension $2 k$. We then have the following principal Spin $(2 k)$ bundle

$$
\operatorname{Spin}(2 k) \rightarrow Q \rightarrow M
$$

which is the double cover of the frame bundle of $T M$, the tangent bundle of $M$. From the two half spinor representations of $\operatorname{Spin}(2 k),\left\{\triangle^{+}, \triangle^{-}\right\}$we get two associated bundles on $M$ which we still denote by $\triangle^{ \pm}$respectively. The Dirac operator on $M$ is a basic elliptic operator between the section space $\Gamma\left(\triangle^{+}\right)$ and $\Gamma\left(\triangle^{-}\right)$. Given another principal bundle $P$ on $M$ with structure group $G$, and any (real) representation $E$ of $G$, we can construct the corresponding associated vector bundle, still denoted by $E$. The Atiyah-Singer index theorem in this case is given by

$$
\text { Ind } D \otimes E=\int_{M} \hat{A}(M) \operatorname{ch} E
$$

where recall that $D \otimes E$ is the twisted Dirac operator

$$
D \otimes E: \Gamma\left(\triangle^{+} \otimes E\right) \rightarrow \Gamma\left(\triangle^{-} \otimes E\right)
$$

and the index is defined to be

$$
\operatorname{Ind} D \otimes E=\operatorname{dim} \operatorname{Ker} D \otimes E-\operatorname{dim} \text { Coker } D \otimes E .
$$

If $\left\{ \pm x_{j}\right\}$ and $\left\{ \pm y_{j}\right\}$ are the formal Chern roots of $T M \otimes C$ and $E \otimes C$ respectively, then

$$
\hat{A}(M)=\prod_{j=1}^{k} \frac{x_{j}}{2 \sinh x_{j} / 2}, \operatorname{ch} E=\sum_{j=1}^{l} e^{y_{j}}+e^{-y_{j}}
$$

are respectively the $\hat{A}$-class of $M$ and the Chern character of $E$.

Another important elliptic operator, the signature operator $d_{s}$ is obtained by taking $E$ to be $\triangle=\Delta^{+} \oplus \triangle^{-}$. We obviously have

where

$$
\text { Ind } D=\int_{M} \hat{A}(M), \text { and Ind } d_{s}=\int_{M} L(M)
$$




$$
L(M)=\prod_{j=1}^{k} \frac{x_{j}}{\tanh x_{j} / 2}
$$

is the Hirzebruch $L$-class of $M$.

As one can see, the starting point of the index formula is the representation theory of spin groups. More precisely, the Chern character of a bundle is induced from the character of the corresponding representation through transgression as in $[\mathrm{BH}]$. Since

$$
\operatorname{ch}\left(\triangle^{+}-\triangle^{-}\right)=\prod_{j=1}^{k}\left(e^{x_{j} / 2}-e^{-x_{j} / 2}\right)=2 \prod_{j=1}^{k} \sinh x_{j} / 2,
$$

the $\hat{A}$-class is essentially the ratio of the Euler class and the Chern character of the basic element $\triangle^{+}-\triangle^{-}$, i.e.

$$
\hat{A}(M)=\frac{e(M)}{\operatorname{ch}\left(\triangle^{+}-\triangle^{-}\right)} .
$$

Most interestingly the denominator of the $\hat{A}$-class can also be viewed as induced from the Weyl denominator in the representation theory of compact Lie groups.

1.2. Loop groups and index theory. In the following, we will show that, if we replace the representations of the classical Lie groups by their correponding loop groups, we exactly recover the complete theory of elliptic genus. For this we need the following simple construction.

Given a principal $G$-bundle $P$ on $M$, and a positive energy representation $E$ of $\tilde{L} G$ which is the central extension of the loop group of $G$, we decompose $E$ according to the rotation action of the loop to get $E=\sum_{>0} E_{n}$ where each $E_{n}$ is a finite dimensional representation of $G$. First assume that $E$ is irreducible. Construct associated bundles to $P$ from each $E_{n}$, which we still denote by $E_{n}$, we get an element

$$
\psi(P, E)=q^{m_{\Lambda}} \sum_{n} E_{n} q^{n}
$$

where $q=e^{2 \pi i \tau}$ with $\tau$ in the upper half plane and $m_{\Lambda}$ is a rational number which is the so-called modular anomaly of the representation $E$, see $[\mathrm{K}]$. This construction extends linearly. If $E=\oplus_{j} E^{j}$, then $\psi(P, E)=\sum_{j} \psi\left(P, E^{j}\right)$.

For any positive integer $l$, the loop group $\tilde{L} \operatorname{Spin}(2 l)$ has four irreducible level 1 positive energy representations. Let us denote them by $S^{+}, S^{-}$and $S_{+}, S_{-}$ respectively. Let $\left\{ \pm \alpha_{j}\right\}$ be the roots of $\operatorname{Spin}(2 l)$. Then the normalized KacWeyl characters of these four representations can be expressed in terms of the 
four Jacobi theta-functions and the Dedekind eta-function [Ch] as follows,

$$
\begin{gathered}
\chi_{S^{+}-S^{-}}=\prod_{j=1}^{l} \frac{\theta\left(\alpha_{j}, \tau\right)}{\eta(\tau)} \quad, \quad \chi_{S_{+}-S_{-}}=\prod_{j=1}^{l} \frac{\theta_{2}\left(\alpha_{j}, \tau\right)}{\eta(\tau)}, \\
\chi_{S^{+}+S^{-}}=\prod_{j=1}^{l} \frac{\theta_{1}\left(\alpha_{j}, \tau\right)}{\eta(\tau)} \quad, \quad \chi_{S_{+}+S_{-}}=\prod_{j=1}^{l} \frac{\theta_{3}\left(\alpha_{j}, \tau\right)}{\eta(\tau)} .
\end{gathered}
$$

Note that here we view $\pi i v$ in the theta-functions in [Ch] as one variable $v$. That is, for example

$$
\theta(v, \tau)=-q^{1 / 8} 2 i \sinh v / 2 \prod_{n=1}^{\infty}\left(1-q^{n}\right)\left(1-e^{v} q^{n}\right)\left(1-e^{-v} q^{n}\right)
$$

It turns out that $S^{+} \pm S^{-}$are the loop group analogues of the finite dimensional spinor representations $\Delta^{+} \pm \Delta^{-}$. Recall that each representation of loop group $\tilde{L} G$ is induced from a representation of $G$. We note that $S^{ \pm}$are induced from $\triangle^{ \pm}$respectively. Let $Q$ be the principal spin bundle of $M$ as in $\S 1.1$. For a vector bundle $V$ on $M$, let

$$
\begin{gathered}
\Lambda_{t} V=1+t \Lambda^{1} V+t^{2} \Lambda^{2} V+\cdots, \\
S_{t} V=1+t S^{1} V+t^{2} S^{2} V+\cdots
\end{gathered}
$$

be the two operations in $K(M)[[t]]$. It is easy to get the following

$$
\begin{aligned}
& \psi\left(Q, S^{-}-S^{-}\right)=q^{-\frac{k}{12}}\left(\triangle^{+}-\triangle^{-}\right) \otimes_{j=1}^{\infty} \Lambda_{-q^{j}}(T M), \\
& \psi\left(Q, S^{+}+S^{-}\right)=q^{-\frac{k}{12}}\left(\triangle^{+}+\triangle^{-}\right) \otimes_{j=1}^{\infty} \Lambda_{q^{j}}(T M) ; \\
& \psi\left(Q, S_{+}-S_{-}\right)=q^{-\frac{k}{24}} \otimes_{j=1}^{\infty} \Lambda_{-q^{j-1 / 2}}(T M) \\
& \psi\left(Q, S_{+}+S_{-}\right)=q^{-\frac{k}{24}} \otimes_{j=1}^{\infty} \Lambda_{q^{j-1 / 2}}(T M) .
\end{aligned}
$$

Just as $\triangle^{+}-\triangle^{-}$induces the Dirac operator on $M, S^{+}-S^{-}$also induces the Dirac operator on $L M$. Similar to the above $\hat{A}$-calss of $M$, introduce the loop space $\hat{A}$-class $\hat{\Theta}(M)$ as

$$
\hat{\Theta}(M)=\frac{e(M)}{\operatorname{ch}\left(\psi\left(Q, S^{+}\right)-\psi\left(Q, S^{-}\right)\right)}=\eta(\tau)^{k} \cdot \prod_{j=1}^{k} \frac{x_{j}}{\theta\left(x_{j}, \tau\right)} .
$$

Then we have similarly the Dirac operator for loop space

$$
D^{L}=q^{-\frac{k}{12}} D \otimes \otimes_{j=1}^{\infty} S_{q^{j}}(T M)
$$

and the corresponding index formula

$$
\text { Ind } D^{L}=\int_{M} \hat{\Theta}(M) \text {. }
$$


A general index theorem in this loop group setting is

$$
\text { Ind } D^{L} \otimes \psi(P, E)=\int_{M} \hat{\Theta}(M) \operatorname{ch} \psi(P, E) .
$$

Especially take $P=Q$ as the principal Spin(2k)-bundle of $M$ and $E=$ $S^{+}+S^{-}$, we get the signature of the loop space $L M$

$$
\text { Ind } D^{L} \otimes \psi\left(Q, S^{+}+S^{-}\right)=\int_{M} \prod_{j=1}^{k} x_{j} \frac{\theta_{1}\left(x_{j}, \tau\right)}{\theta\left(x_{j}, \tau\right)} \text {. }
$$

We call

$$
\prod_{j=1}^{k} x_{j} \frac{\theta_{1}\left(x_{j}, \tau\right)}{\theta\left(x_{j}, \tau\right)}
$$

the elliptic $L$-class.

Associated to $S_{+} \pm S_{-}$are the other two elliptic operators which do not have finite dimensional analogues. Their indice are

$$
\begin{aligned}
& \text { Ind } D^{L} \otimes \psi\left(Q, S_{-}-S_{-}\right)=\int_{M} \prod_{j=1}^{k} x_{j} \frac{\theta_{2}\left(x_{j}, \tau\right)}{\theta\left(x_{j}, \tau\right)}, \\
& \text { Ind } D^{L} \otimes \psi\left(Q, S_{-}+S_{-}\right)=\int_{M} \prod_{j=1}^{k} x_{j} \frac{\theta_{3}\left(x_{j}, \tau\right)}{\theta\left(x_{j}, \tau\right)} .
\end{aligned}
$$

It is just because of these two new elliptic operators that make the proof of the famous Witten rigidity theorems very simple. This is the magic of infinite dimensional geometry and topology.

1.3. Elliptic genera. The indices of the above three elliptic operators are actually modular functions. To get modular forms instead, we consider their virtual versions. This means that we replace $T M$ by its virtual bundle $\bar{T} M=$ $T M-2 k$ in the above symmetric and wedge products. Denote by

$$
\mathfrak{D}^{L}=D \otimes \otimes_{j=1}^{\infty} S_{q^{j}}(\bar{T} M),
$$

we get

$$
\operatorname{Ind} \mathfrak{D}^{\mathfrak{L}}=\int_{\mathfrak{M}} \prod_{\mathfrak{j}=1}^{\mathfrak{k}} \mathfrak{x}_{\mathfrak{j}} \frac{\theta^{\prime}(\mathfrak{o}, \tau)}{\theta\left(\mathfrak{x}_{\mathfrak{j}}, \tau\right)}
$$

which is called the Witten genus; and

$$
\operatorname{Ind} \mathfrak{D}^{\mathfrak{L}} \otimes \triangle \otimes_{\mathfrak{m}=\mathbf{1}}^{\infty} \Lambda_{\mathfrak{q}^{\mathfrak{m}}}(\overline{\mathfrak{T}} \mathfrak{M})=\int_{\mathfrak{M}} \prod_{\mathfrak{j}=1}^{\mathfrak{k}} \mathfrak{x}_{\mathfrak{j}} \frac{\theta^{\prime}(\mathfrak{o}, \tau) \theta_{\mathfrak{1}}\left(\mathfrak{x}_{\mathfrak{j}}, \tau\right)}{\theta\left(\mathfrak{x}_{\mathfrak{j}}, \tau\right) \theta_{\mathbf{1}}(\mathfrak{o}, \tau)}
$$




$$
\begin{aligned}
\operatorname{Ind} \mathfrak{D}^{\mathfrak{L}} \otimes_{\mathfrak{m}=\mathbf{1}}^{\infty} \Lambda_{-\mathfrak{q}^{\mathfrak{m}-1 / 2}}(\overline{\mathfrak{T}} \mathfrak{M}) & =\int_{\mathfrak{M}} \prod_{\mathfrak{j}=1}^{\mathfrak{k}} \mathfrak{x}_{\mathfrak{j}} \frac{\theta^{\prime}(\mathfrak{o}, \tau) \theta_{2}\left(\mathfrak{x}_{\mathfrak{j}}, \tau\right)}{\theta\left(\mathfrak{x}_{\mathfrak{j}}, \tau\right) \theta_{2}(\mathfrak{o}, \tau)} \\
\operatorname{Ind} \mathfrak{D}^{\mathfrak{L}} \otimes_{\mathfrak{m}=\mathbf{1}}^{\infty} \Lambda_{\mathfrak{q}^{\mathfrak{m}-1 / 2}}(\overline{\mathfrak{T}} \mathfrak{M}) & =\int_{\mathfrak{M}} \prod_{\mathfrak{j}=1}^{\mathfrak{k}} \mathfrak{x}_{\mathfrak{j}} \frac{\theta^{\prime}(\mathfrak{o}, \tau) \theta_{3}\left(\mathfrak{x}_{\mathfrak{j}}, \tau\right)}{\theta\left(\mathfrak{x}_{\mathfrak{j}}, \tau\right) \theta_{3}(\mathfrak{o}, \tau)}
\end{aligned}
$$

which are usually called universal elliptic genera. The three functions, suitably normalized, appeared in the above index formulas,

$$
F_{j}(x)=\frac{1}{2 i} \frac{\theta_{j}(x, \tau) \theta^{\prime}(0, \tau)}{\theta(x, \tau) \theta_{j}(0, \tau)}
$$

with $j=1,2,3$ are called their generating series correspondingly. Also we simply call

$$
\prod_{j=1}^{k} x_{j} \frac{\theta^{\prime}(0, \tau)}{\theta\left(x_{j}, \tau\right)}
$$

the Witten class and write it as $W(M)$. Obviously

$$
W(M)=\eta(\tau)^{2 k} \hat{\Theta}(M) .
$$

Similarly

$$
\prod_{j=1}^{k} x_{j} \frac{\theta^{\prime}(0, \tau) \theta_{1}\left(x_{j}, \tau\right)}{\theta\left(x_{j}, \tau\right) \theta_{1}(0, \tau)}
$$

is called the normalized elliptic $L$-class of $M$.

It is worthwhile to record the following remarks. Assume there exists a torus action on a manifold $M$ with fixed point set $F$ and normal bundle $N$. Then according to the action, $N$ can be decomposed into sums of complex line bundles $\left\{L_{j}\right\}$ and the torus acts on $L_{j}$ by $g^{m_{j}}$, where $g$ is a generator of the torus and $m_{j}$ is called the weight of $L_{j}$.

The equivariant Euler class of $N$ is by definition $\prod_{j}\left(m_{j}+x_{j}\right)$ where $x_{j}$ is the first Chern class of $L_{j}$. We define the normalized equivariant Euler class of $N$ to be $\prod_{j}\left(1+x_{j} / m_{j}\right)$.

Let $L M$ denote the loop space of $M$ and $L L M$ the double loop space of $M$. The rotation of the circles induces a natural circle action on $L M$ and an $S^{1} \times S^{1}$ action on $L M$ with fixed points $M$. The normal bundle $N_{L}$ of $M$ in $L M$ is $\oplus_{n \neq 0} T M_{n}$ where each $T M_{n}$ is the same as $T M$ with weight $n$. The normal bundle $N_{L L}$ of $M$ in $L L M$ is $\oplus_{n, m \neq 0} T M_{n, m}$ with $T M_{n, m}$ the same as $T M$ with weight $n+m \tau$.

As a simple consequence of Eisenstein's product formulas for sine function and theta-function [We], we have the following 
1) the normalized equivariant Euler class of $N_{L}$ is the inverse of the $\hat{A}$-class of $M$.

2) the normalized equivariant Euler calss of $N_{L L}$ is the inverse of the $\hat{\Theta}$-class of $M$.

In certain sense we can say that the classical Hirzebruch genera, such as $\hat{A}$-genus and $L$-genus, are 1-periodic genera, since they are associated to triognometric functions; while the elliptic genera, associated to elliptic functions, are 2-periodic genera. Note that the first observation above is due to Witten. From a pure functional theoretic point of view, make the following replacement in the Hirzebruch $\hat{A}$-class and $L$-class,

$$
\begin{aligned}
\sinh x / 2 & \rightarrow \frac{\theta(x, \tau)}{\eta(\tau)} \\
\cosh x / 2 & \rightarrow \frac{\theta_{1}(x, \tau)}{\eta(\tau)},
\end{aligned}
$$

one exactly recovers the $\hat{\Theta}$-class and the elliptic $L$-calss. On the other hand, make the following replacement

$$
\begin{aligned}
& \sinh x / 2 \rightarrow \frac{\theta(x, \tau)}{\theta^{\prime}(0, \tau)} \\
& \cosh x / 2 \rightarrow \frac{\theta_{1}(x, \tau)}{\theta_{1}(0, \tau)},
\end{aligned}
$$

we get the Witten class and the normalized elliptic $L$-class.

1.4. Elliptic genera and theta-functions. Let $\mathfrak{P}(\mathfrak{x})$ be the Weierstrass elliptic function associated to the lattice $\{2 m \pi+2 n \pi \tau\}$. Then one has the following parametrization of elliptic curves

$$
\mathfrak{P}^{\prime}(\mathfrak{x})=4\left(\mathfrak{P}(\mathfrak{x})-\mathfrak{e}_{1}\right)\left(\mathfrak{P}(\mathfrak{x})-\mathfrak{e}_{2}\right)\left(\mathfrak{P}(\mathfrak{x})-\mathfrak{e}_{3}\right)
$$

with $e_{j}=\mathfrak{P}\left(\omega_{\mathrm{j}}\right)$ where $\omega_{1}=\pi, \omega_{2}=\pi \tau, \omega_{3}=\pi(1+\tau)$.

The interesting connection of elliptic genera with elliptic curves are manifested by the following relations, for $j=1,2,3$

$$
2 i F_{j}(x)=\sqrt{\mathfrak{P}(\mathfrak{x})-\mathfrak{e}_{\mathfrak{j}}}
$$

which is easily seen by comparing poles of the functions of both sides.

For $j=1,2,3$, let us denote $\theta_{j}(0, \tau)$ by $\theta_{j}$, we then have relations

$$
e_{3}-e_{2}=\theta_{1}^{4}, e_{1}-e_{3}=\theta_{2}^{4}, e_{1}-e_{2}=\theta_{3}^{4} .
$$

Here note that we use $v$, instead of $\pi v$ as the variable in the theta-functions in [Ch]. This is also slightly different from $§ 1.2$. This change is only for convenience and does not matter much. Since $\mathfrak{P}^{\prime}(\mathfrak{x})=-8 \mathfrak{F}_{\mathfrak{j}}(\mathfrak{x}) \mathfrak{F}_{\mathfrak{j}}^{\prime}(\mathfrak{x})$, plug into 
the Weierstrass equation, we get the functional equations of the three elliptic genera.

$$
\begin{aligned}
& F_{1}(x)^{2}=\left(F_{1}(x)^{2}-1 / 4 \theta_{3}^{4}\right)\left(F_{1}(x)^{2}-1 / 4 \theta_{2}^{4}\right), \\
& F_{2}(x)^{2}=\left(F_{2}(x)^{2}+1 / 4 \theta_{3}^{4}\right)\left(F_{2}(x)^{2}+1 / 4 \theta_{1}^{4}\right), \\
& F_{3}(x)^{2}=\left(F_{3}(x)^{2}+1 / 4 \theta_{2}^{4}\right)\left(F_{3}(x)^{2}-1 / 4 \theta_{1}^{4}\right) .
\end{aligned}
$$

¿From these equations, we easily get the logrithms of the three elliptic genera

$$
\begin{aligned}
& g_{1}(x)=\int_{0}^{x} \frac{d u}{\sqrt{\left(1-1 / 4 \theta_{3}^{4} u^{2}\right)\left(1-1 / 4 \theta_{2}^{4} u^{2}\right)}}, \\
& g_{2}(x)=\int_{0}^{x} \frac{d u}{\sqrt{\left(1+1 / 4 \theta_{3}^{4} u^{2}\right)\left(1+1 / 4 \theta_{1}^{4} u^{2}\right)}}, \\
& g_{3}(x)=\int_{0}^{x} \frac{d u}{\sqrt{\left(1+1 / 4 \theta_{2}^{4} u^{2}\right)\left(1-1 / 4 \theta_{1}^{4} u^{2}\right)}} .
\end{aligned}
$$

In fact, let $z$ denote the first Chern class of the universal line bundle on $C P^{n}$. By definition,

$$
g_{j}(x)=\sum_{n=0}^{\infty} \varphi_{j}\left(C P^{2 n}\right) \frac{x^{2 n+1}}{2 n+1}, j=1,2,3,
$$

where $\varphi_{j}$ denotes the corresponding elliptic genus associated to $x F_{j}(x)$. Since

$$
\varphi_{j}\left(C P^{2 n}\right)=\int_{C P^{2 n}}\left(z F_{j}(z)\right)^{2 n+1}=\frac{1}{n !} \frac{d^{n}}{d z^{n}}\left[z F_{j}(z)\right]^{2 n+1},
$$

it is easy to show, by Lagrange theorem [Co], that

$$
g_{j}^{-1}(x)=\frac{1}{F_{j}(x)} .
$$

Compare with the Ochanine's standard equation for elliptic genus,

$$
y^{2}=1-2 \delta x^{2}+\varepsilon x^{4},
$$

we have the following expressions,

$$
\begin{gathered}
\delta_{1}=1 / 8\left(\theta_{2}^{4}+\theta_{3}^{4}\right), \varepsilon_{1}=1 / 16 \theta_{2}^{4} \theta_{3}^{4}, \\
\delta_{2}=-1 / 8\left(\theta_{1}^{4}+\theta_{3}^{4}\right), \varepsilon_{2}=1 / 16 \theta_{1}^{4} \theta_{3}^{4}, \\
\delta_{3}=1 / 8\left(\theta_{2}^{4}-\theta_{2}^{4}\right), \varepsilon_{3}=-1 / 16 \theta_{1}^{4} \theta_{2}^{4} .
\end{gathered}
$$


The $\delta_{j}$ 's are modular forms of level 2 and the $\varepsilon_{j}$ 's are modular forms of level 4 , as easily follow from the properties of theta-functions. Note that these formulas give us the infinite product expansions of the $\varepsilon$ 's. They are very useful in following discussions. These three elliptic genera are uniquely characterized by their functional equations, as well as by their topological properties, such as rigidity under group action and multiplicativaty under spin fibrations. See [HBJ], [O], [S] and below for further discussions.

\section{Applications in topology}

The combining strength of index theory, Kac-Moody algebras, elliptic functions and modular forms in the above discussion can help us get many interesting topological results. Here for simplicity, I will only explain several special examples. For more general discussion see [Liu1]. The following two lists may be helpful in understanding the general picture of applications. First many results on a compact smooth spin manifold $M$ have analogues on its loop space $L M$

$$
\begin{array}{rll}
M & --- & L M \\
\hat{A}-\text { vanishing theorem } & -- & \hat{\Theta}-\text { vanishing theorem } \\
d_{s} \text { is rigid } & -- & \text { the Witten rigidity theorem } \\
\text { signature } \equiv 0(\bmod 16) & -- & \text { signature } \equiv 0(\bmod 16) .
\end{array}
$$

But the results on $L M$ are much more stronger and many of them have no finite dimensional analogues. More precisely we have the following list

$$
\begin{array}{rll}
M & --- & L M \\
? & -- & \text { higher level rigidity theorems } \\
? & -- & \text { higher level vanishing theorems } \\
? & -- & \text { general miraculous cancellation. }
\end{array}
$$

Here higher level means higher level loop group representation which appears natrually in our study. Of course one can get results on $M$ by specializing the results on $L M$. We hope quantum group, which is the finite dimensional counterpart of affine Lie algebra, may eventually help explain the above ?'s.

2.1. Miraculous cancellation formula. In $[\mathrm{AW}]$, a gravitational anomaly cancellation formula was derived from direct computations. This is a formula relating the $L$-class to the $\hat{A}$-class and a twisted $\hat{A}$-class of a 12 -dimensional manifold. More precisely, let $M$ be a smooth manifold of dimension 12, then the miraculous cancellation formula is

$$
L(M)=8 \hat{A}(M, T)-32 \hat{A}(M)
$$


where $T=T M$ denotes the tangent bundle of $M$ and the equality holds at the top degree of each differential form. Here

$$
\hat{A}(M, T)=\hat{A}(M) \operatorname{ch} T
$$

is the $\hat{A}$-class twisted by tangent bundle.

Using elliptic genera, we can easily derive a much general formula for manifolds of any dimension. We can even get a formula with a general vector bundle involved. For simplicity, let us just take a manifold $M$ of dimension $8 k+4$ and only consider the tangent bundle case. Write

$$
\begin{gathered}
\Theta_{1}(M)=\otimes_{j=1}^{\infty} S_{q^{j}}(\bar{T} M) \otimes \otimes_{m=1}^{\infty} \Lambda_{q^{m}}(\bar{T} M), \\
\Theta_{2}(M)=\otimes_{j=1}^{\infty} S_{q^{j}}(\bar{T} M) \otimes \otimes_{m=1}^{\infty} \Lambda_{-q^{m-1 / 2}}(\bar{T} M) .
\end{gathered}
$$

They have the following expansions in $q$ with coefficients in $K(M)$

$$
\begin{gathered}
\Theta_{1}(M)=A_{0}+A_{1} q+\cdots, \\
\Theta_{2}(M)=B_{0}+B_{1} q^{\frac{1}{2}}+\cdots .
\end{gathered}
$$

Denote the top degree terms in

$$
L(M) \operatorname{ch} \Theta_{1}(M) \text { and } \hat{A}(M) \operatorname{ch} \Theta_{2}(M)
$$

by $P_{1}(\tau)$ and $P_{2}(\tau)$ respectively.

Let $\Gamma_{0}(2), \Gamma^{0}(2)$ be the modular subgroups,

$$
\begin{aligned}
& \Gamma_{0}(2)=\left\{\left(\begin{array}{ll}
a & b \\
c & d
\end{array}\right) \in S L_{2}(Z) \mid c \equiv 0(\bmod 2)\right\} \\
& \Gamma^{0}(2)=\left\{\left(\begin{array}{ll}
a & b \\
c & d
\end{array}\right) \in S L_{2}(Z) \mid b \equiv 0(\bmod 2)\right\} .
\end{aligned}
$$

Recall that a modular form over a modular subgroup $\Gamma$ is a holomorphic function $f(\tau)$ defined on the upper half plane $H$ such that for any $g=\left(\begin{array}{ll}a & b \\ c & d\end{array}\right) \in$ $\Gamma$, one has

$$
f\left(\frac{a \tau+b}{c \tau+d}\right)=\chi(g)(c \tau+d)^{k} f(\tau)
$$

where $\chi: \Gamma \rightarrow C^{*}$ is a character on $\Gamma$ and $k$ is called the weight of $f$. We also assume that $f$ is holomorphic at $\tau=i \infty$. The following lemma can be proved by using the theta-function expressions of $P_{1}(\tau)$ and $P_{2}(\tau)$ as given in $\S 1$.

Lemma 2.1.1. $P_{1}(\tau)$ is a modular form of weight $4 k+2$ over $\Gamma_{0}(2) ; P_{2}(\tau)$ is a modular form of weight $4 k+2$ over $\Gamma^{0}(2)$. 
Let

$$
\begin{aligned}
\delta_{1}(\tau)=\frac{1}{8}\left(\theta_{2}^{4}+\theta_{3}^{4}\right) & , & \varepsilon_{1}(\tau)=\frac{1}{16} \theta_{2}^{4} \theta_{3}^{4}, \\
\delta_{2}(\tau)=-\frac{1}{8}\left(\theta_{1}^{4}+\theta_{3}^{4}\right) & , & \varepsilon_{2}(\tau)=\frac{1}{16} \theta_{1}^{4} \theta_{3}^{4}
\end{aligned}
$$

be as in $§ 1.4$. They have the following Fourier expansions in $q$

$$
\begin{array}{ccc}
\delta_{1}(\tau)=\frac{1}{4}+6 q+\cdots & , \quad \varepsilon_{1}(\tau)=\frac{1}{16}-q+\cdots, \\
\delta_{2}(\tau)=-\frac{1}{8}-3 q^{\frac{1}{2}}+\cdots, & \varepsilon_{2}(\tau)=q^{\frac{1}{2}}+\cdots,
\end{array}
$$

where "..." are the higher degree terms all of which are of integral coefficients.

Let $M(\Gamma)$ denote the ring of modular forms over $\Gamma$ with integral Fourier coefficients.

Using the transformation formulas of the Jacobi theta-functions [Ch], we get

Lemma 2.1.2. $\delta_{1}, \delta_{2}$ are modular forms of weight 2 and $\varepsilon_{1}, \varepsilon_{2}$ are modular forms of weight 4 , and furthermore $M\left(\Gamma^{0}(2)\right)=Z\left[8 \delta_{2}(\tau), \varepsilon_{2}(\tau)\right]$.

In view of these two Lemmas we can write

$$
P_{2}(\tau)=b_{0}\left(8 \delta_{2}\right)^{2 k+1}+b_{1}\left(8 \delta_{2}\right)^{2 k-1} \varepsilon_{2}+\cdots+b_{k}\left(8 \delta_{2}\right) \varepsilon_{2}^{k},
$$

where the $b_{j}$ 's are integral linear combinations of the top degree terms of the $\left\{\hat{A}(M) \operatorname{ch} B_{j}\right\}$ 's.

Apply the modular transformation $S: \tau \rightarrow-\frac{1}{\tau}$, we have

$$
\begin{array}{r}
\delta_{2}\left(-\frac{1}{\tau}\right)=\tau^{2} \delta_{1}(\tau) \quad, \quad \varepsilon_{2}\left(-\frac{1}{\tau}\right)=\tau^{4} \varepsilon_{1}(\tau), \\
P_{2}\left(-\frac{1}{\tau}\right)=2^{-(4 k+2)} \tau^{4 k+2} P_{1}(\tau) .
\end{array}
$$

Therefore,

$$
P_{1}(\tau)=2^{4 k+2}\left[b_{0}\left(8 \delta_{1}\right)^{2 k+1}+b_{1}\left(8 \delta_{1}\right)^{2 k-1} \varepsilon_{1}+\cdots+b_{k}\left(8 \delta_{1}\right) \varepsilon_{1}^{k}\right] .
$$

At $q=0,8 \delta_{1}=2$ and $\varepsilon_{1}=2^{-4}$. We get a special case of the general miraculus cancellation formula,

Theorem 2.1. At top degree, the following identity holds,

$$
L(M)=2^{3} \sum_{j=0}^{k} 2^{6 k-6 j} b_{j} .
$$

For a more general formula, see [Liu1]. Denote by $b_{j}(T M)$ the integral linear combinations of the $B_{i}(T M)$ 's which gives the polynomials $b_{j}$ in the theorem. It is rather clear that these $b_{j}(T M)$ 's can be determined canonically. 
We have, for a compact oriented smooth manifold $M$,

Corollary 2.1. The follwoing identity holds,

$$
\operatorname{Sign}(M)=2^{3} \sum_{j=0}^{k} 2^{6 k-6 j} \int_{M} \hat{A}(M) \operatorname{ch} b_{j}(T M) .
$$

The left hand side denotes the signature of $M$. In particular, if $M$ is spin, then each characteristic number on the right hand side is an even integer. One thus recovers the Ochanine theorem [O1],

Corollary 2.2. The signature of an $8 k+4$ dimensional compact spin manifold is divisible by 16 .

Actually the proof shows that $P_{1}(\tau)$, the signature of the corresponding loop space $L M$ is divisible by 16 . Combining with the Atiyah-Patodi-Singer index formula, the above miraculous cancellation formula gives us interesting analytic expressions of some topological invariants. It can also be used to express the holonomy of certain determinant line bundles in terms of $\eta$-invariants. See [Liu1] and [LZ] for more details. Note that the main idea of the above proof are due to Hirzebruch [H1] and Landweber [La].

2.2. Rigidity. Elliptic genus originated from many people's trying to find the generating series of rigid elliptic operators. Given a smooth compact manifold with an action of a group $G$. Let $P$ be an elliptic operator on $M$ which commutes with the action. Then both the kernal and the cokernal of $P$ are finite dimensional representations of $G$. The Lefschetz number of $P$ at $g \in G$ is defined to be

$$
L_{P}(g)=\operatorname{Tr}_{g} \operatorname{Ker} P-\operatorname{Tr}_{g} \text { Coker } P
$$

which is a character of $G$.

We say $P$ is rigid with respect to $G$, if $L(g)$ is independent of $g$, that is a constant character of $G$. To prove the rigidity of an elliptic operator with respect to a general compact connected Lie group action, we obviously only need to study its rigidity with respect to $S^{1}$-action which will be our only concern in the following.

The rigidity of the signature operator $d_{s}$ with respect to $S^{1}$-action on $M$ is a trivial fact, since its kernal and cokernal are contained in the deRham cohomology. If $M$ is spin, the rigidity of the Dirac operator with respect to the $S^{1}$-action is the famous $\hat{A}$-vanishing theorem of $[\mathrm{AH}]$. As will be seen in the following, the rigidity of the signature operator with respect to $S^{1}$ action on loop space was conjectured by Witten and is highly non-trivial. The correponding $\hat{A}$-vanishing theorem for loop space will be discussed in next section. 
In [W] Witten first conjectured the rigidity of $D \otimes T M$ and proved it for compact homogeneous spin manifolds. In trying to prove it in general and to find other rigid elliptic operators, Landweber-Stong and Ochanine discovered elliptic genus [LS], [O]. Witten, motivated by quantum field theory, conjectured that all of the three elliptic operators associted to the three elliptic genera, i.e.

$$
\begin{aligned}
& \mathfrak{D}^{\mathfrak{L}} \otimes \triangle \otimes_{\mathfrak{j}=1}^{\infty} \Lambda_{\mathfrak{q}^{\mathfrak{j}}}(\overline{\mathfrak{T}} \mathfrak{M}) \\
& \mathfrak{D}^{\mathfrak{L}} \otimes \otimes_{\mathfrak{j}=1}^{\infty} \Lambda_{-\mathfrak{q}^{\mathfrak{j}-1 / 2}}(\overline{\mathfrak{T}} \mathfrak{M}) \\
& \mathfrak{D}^{\mathfrak{L}} \otimes \otimes_{\mathfrak{j}=1}^{\infty} \Lambda_{\mathfrak{q}^{\mathfrak{j}-1 / 2}}(\overline{\mathfrak{T}} \mathfrak{M})
\end{aligned}
$$

are rigid. Note that, so far no body has been able to give a direct proof of the rigidity of $D \otimes T M$ for a general spin manifold $M$ which is only known through the following theorem.

Theorem 2.2. The above three elliptic operators are rigid.

This means that, if we expand the above elliptic operators into formal power series in $q$, for example

$$
\mathfrak{D}^{\mathfrak{L}} \otimes \otimes_{\mathfrak{m}=\mathbf{1}}^{\infty} \Lambda_{\mathfrak{q}^{\mathfrak{m}-1 / 2}}(\overline{\mathfrak{T}} \mathfrak{M})=\sum_{\mathfrak{j}=1}^{\infty} \mathfrak{D} \otimes \mathfrak{E}_{\mathfrak{j}} \mathfrak{q}^{\mathfrak{j}-1 / 2},
$$

then each $D \otimes E_{j}$ is rigid. Note that $D \otimes T M$ is the second term.

This theorem was first proved in $[\mathrm{T}],[\mathrm{BT}]$. Our proof uses the key properties of the Jacobi theta-functions and the Atiyah-Bott-Segal-Singer fixed point formula. For simplicity we only consider the isolated fixed point situation. Let $\{p\} \subset M$ be the fixed points of a generator $g=e^{2 \pi i t} \in S^{1}$. Let $\left\{m_{j}\right\}$ be the exponents of $T M$ at the fixed point $p$. That is, we have orientationcompatible decompositions

$$
\left.T M\right|_{p}=E_{1} \oplus \cdots \oplus E_{k}, k=\frac{1}{2} \operatorname{dim} M,
$$

and $g$ acts on $E_{j}$ by $g^{m_{j}}$.

let us denote the Lefschetz numbers of the above three elliptic operators by

$$
F_{1}(t, \tau), F_{2}(t, \tau) \text { and } F_{3}(t, \tau)
$$

respectively. Apply the Lefschetz fixed point formula $[\mathrm{AB}]$, we have

$$
\begin{aligned}
& F_{1}(t, \tau)=(2 \pi i)^{-k} \sum_{p} \prod_{j=1}^{k} \frac{\theta_{1}\left(m_{j} t, \tau\right) \theta^{\prime}(0, \tau)}{\theta\left(m_{j} t, \tau\right) \theta_{1}(0, \tau)}, \\
& F_{2}(t, \tau)=(2 \pi i)^{-k} \sum_{p} \prod_{j=1}^{k} \frac{\theta_{2}\left(m_{j} t, \tau\right) \theta^{\prime}(0, \tau)}{\theta\left(m_{j} t, \tau\right) \theta_{2}(0, \tau)},
\end{aligned}
$$




$$
F_{3}(t, \tau)=(2 \pi i)^{-k} \sum_{p} \prod_{j=1}^{k} \frac{\theta_{3}\left(m_{j} t, \tau\right) \theta^{\prime}(0, \tau)}{\theta\left(m_{j} t, \tau\right) \theta_{3}(0, \tau)} .
$$

Here the theta-functions are the same as in $[\mathrm{Ch}]$. The rigidity is equivalent to the fact that the above $F$ 's are independent of $t$. First we can obviously extend these $F$ 's to well-defined meromorphic functions on $(t, \tau) \in \mathbf{C} \times \mathbf{H}$. The key point is to show that they are actually holomorphic. The proof of the theorem is divided into three steps.

Lemma 2.2.1. The $F$ 's are doubly periodic on the lattice $\{n+m \tau\}$.

That is we have $F_{j}(t+n+m \tau)=F_{j}(t, \tau)$. For $g=\left(\begin{array}{ll}a & b \\ c & d\end{array}\right) \in S L_{2}(Z)$, define modular transformation of $g$ on the $F$ 's as

$$
F(g(t, \tau))=(c \tau+d)^{-k} F\left(\frac{t}{c \tau+d}, \frac{a \tau+b}{c \tau+d}\right) .
$$

Then we have

Lemma 2.2.2. The integral span of the three F's are invariant under the action of $S L_{2}(Z)$.

These two lemmas can be proved by using the transformation formulas of the four Jacobi theta-functions [Ch]. It depends essentially on the 'elliptic property' of these elliptic genera.

Lemma 2.2.3. The three $F$ 's are holomorphic for $t$ real and $\tau$ in upper half plane.

Note that $t$ real implies that $e^{2 \pi i t}$ lies in $S^{1}$, and that the definition of Lefschetz number imples that it is the character of the action group $S^{1}$, therefore it is holomorphic as a function of $g \in S^{1}$. In our case, these $F$ 's can be expanded as power series in $q$ with coefficients the combinations of the characters of $S^{1}$ representations. The Lefschetz fixed point forumla comes into play crucially at this point.

Then a key observation is that these three lemmas implies the rigidity. In fact, modular transformation, together with lemma 2, transforms the regularity of the $F^{\prime}$ 's on $S^{1}$ to everywhere in the complex plane; the first lemma immediately implies that they are constant. See [Liu0] for the detail.

2.3. A vanishing theorem of the Witten genus. Let $M$ be a compact smooth spin manifold with an $S^{1}$-action, in [AH], Atiyah-Hirzebruch proved the following theorem,

Theorem [AH]: The $\hat{A}$-genus of $M$ is zero.

As a corollary, we know that a compact smooth spin manifold with non-zero $\hat{A}$-genus does not admit any compact connected Lie group action. 
Let $M$ be compact smooth and spin, with an $S^{1}$-action, and $M S^{1}=E S^{1} \times S^{1}$ $M$ be the Borel model of $M$. Let $u$ be the generator of $H^{*}\left(B S^{1}, Z\right)$. Recall that $B S^{1}=C P^{\infty}$ and $E S^{1}=S^{\infty}$. The equivariant characteristic classes of $M$ are defined to be the usual characteristic classes of $M S^{1}$. Let $p_{1}(M)_{S^{1}}$ denote the first equivariant Pontrjagin class with respect to the $S^{1}$-action. Let

$$
\pi: \quad M S^{1} \rightarrow B S^{1}
$$

be the canonical projection and

$$
\pi^{*}: \quad H^{*}\left(B S^{1}, Z\right) \rightarrow H^{*}\left(M S^{1}, Z\right)
$$

be the pull-back in cohomology. Corresponding to the above theorem of Atiyah-Hirzebruch, we have the following theorem for $L M$,

Theorem 2.3. If $p_{1}(M)_{S^{1}}=n \cdot \pi^{*} u^{2}$ for some integer $n$, then the Witten genus of $M$ is zero.

Note that the Witten genus is the $\hat{A}$-genus of $L M$ which is the index of

$$
D \otimes \otimes_{m=1}^{\infty} S_{q^{m}}(\bar{T} M) .
$$

The Witten genus is the virtual version of the $\hat{\Theta}$-genus which is the index of

$$
D \otimes \otimes_{m=1}^{\infty} S_{q^{m}}(T M) .
$$

They differ by a factor $\eta(\tau)^{2 k}$.

¿From quantum field theory, we know that, given a compact smooth manifold $M$, the loop space $L M$ is orientable, if and only if $M$ is spin; $L M$ is spin, if and only if $p_{1}(M)$, the first Pontrjagin class of $M$ is zero. The condition on the first equivariant Pontrjagin class in Theorem 2.3 is equivalent to that $p_{1}(M)=0$ and the $S^{1}$-action preserves this condition, i.e. $L M$ is spin and the $S^{1}$-action preserves this spin structure. Note that in Atiyah-Hirzebruch's situation, one can always lift the action to a double cover to make the action preserve the spin structure of $M$. The reason is that the second StieffelWhitney class whose vanishing is equivalent to the existence of spin structure on $M$ is 2-torsion.

If there is an non-abelian compact connected Lie group action, the condition on $p_{1}(M)_{S^{1}}$ in Theorem 2.3 is equivalent to $p_{1}(M)=0$ which gives us the following corollary due to Dessai [D],

Corollary 2.3. Let $M$ be a compact smooth spin manifold with a non-abelian compact connected Lie group action. If $p_{1}(M)=0$, then the Witten genus of $M$ is zero.

Especially the Witten genus of any compact homogeneous spin manifold with $p_{1}=0$ vanishes. This is the first concrete evidence of the following conjecture of Hoehn and Stolz: 
Conjecture: Any compact smooth spin manifold with $p_{1}=0$ and positive Ricci curvature has vanishing Witten genus.

The proof of our loop space $\hat{A}$-vanishing theorem is a refinement of the proof of the rigidity theorem in last section. Still let us only consider the isolated fixed point case and use the same notation as in last section. The Lefschetz number $H(t, \tau)$ of

is given by

$$
D \otimes \otimes_{m=1}^{\infty} S_{q^{m}}(\bar{T} M)
$$

$$
H(t, \tau)=(2 \pi i)^{-k} \sum_{p} \prod_{j=1}^{k} \frac{\theta^{\prime}(0, \tau)}{\theta\left(m_{j} t, \tau\right)} .
$$

Obviously $H(t, \tau)$ can be extended to $C \times H$ as a meromorphic function.

Lemma 2.3.1. For $m, n \in Z$,

$$
H(t+n+m \tau, \tau)=e^{|n| \pi i(2 t+\tau)} H(t, \tau) .
$$

This tells us the behaviour of $H(t, \tau)$ under the translation of lattice. Its behaviour under modular transformation is given by the following

Lemma 2.3.2. For $g=\left(\begin{array}{ll}a & b \\ c & d\end{array}\right) \in S L_{2}(Z)$, we have

$$
H\left(\frac{t}{c \tau+d}, \frac{a \tau+b}{c \tau+d}\right)=(c \tau+d)^{k} e^{-|n| c t^{2} /(c \tau+d)} H(t, \tau) .
$$

These two lemmas imply that $H(t, \tau)$ is a (meromorphic) Jacobi form of index $-|n| / 2$ and weight $k$. On the other hand, as in Lemma 2.2.3, the Atiyah-Bott-Segal-Singer Lefschetz fixed point formula tells us that $H(t, \tau)$ is holomorphic for $t \in R$ and $\tau$ in the upper half plane which together with the above two lemmas gives us the regularity of $H(t, \tau)$ in $t$ on the whole complex plane. Therefore $H(t, \tau)$ is a holomorphic Jacobi form of negative index which is impossible, except it is zero. Actually it is a simple exercise to prove that the number of zeroes in a fundemental domain of the lattice of a holomorphic Jacobi form is equal to its index [EZ].

2.4. Kac-Moody algebras in topolgy. Let $G$ be a simple and simply connected Lie group, and $\tilde{L} G$ be the central extension of its loop group. Let $E$ be a positive energy representation of $\tilde{L} G$ of level $m$ and $P$ be a principal $G$-bundle on a compact smooth spin manifold $M$. Let $\psi(P, E)$ be the element constructed in $\S 1$ from $P$ and $E$, and assume that there exists an $S^{1}$-action on $M$ which lifts to $P$.

Theorem 2.4. If $p_{1}(M)_{S^{1}}=m \cdot p_{1}(P)_{S^{1}}$, then

$$
D^{L} \otimes \psi(P, E)
$$

is rigid. 
Here $p_{1}(P)_{S^{1}}$ is the equivariant first Pontrjagin class of $P$. The proof of this theorem follows the same idea of the above modular invariance argument. The modular invariance of Kac character formula for the corresponding affine Lie algebras, instead of the classical Jacobi theta-functions comes into play.

Similarly we have the following

Theorem 2.5. If $m \cdot p_{1}(P)_{S^{1}}-p_{1}(M)_{S^{1}}=n \cdot \pi^{*} u^{2}$ with $n$ a negative integer, then the Lefschetz number, especially the index of

$$
D^{L} \otimes \psi(P, E)
$$

vanishes.

We refer to [Liu] and [GL] for the proofs of the above results. It should be interesting to generalize them from affine Lie algebras to vertex operator algebras.

\section{VECTOR BUNDLES AND MODULAR FORMS}

In this section we first introduce infinite dimensional vector bundles on finite dimensional manifolds, and then describe the corresponding $K$-group and Riemann-Roch type theorems. We then construct rings from families of vector bundles with certain modularity restriction. Modulo torsion, these rings give construction of elliptic cohomology in terms of families of vector bundles. Part of the work in this section is still under progress.

3.1. Infinite dimensional vector bundles. Let $M$ be a manifold. By using transition functions [L] we can define an infinite dimensional vector bundle $E$ with Hilbert space as fiber just in the same way as define a finite dimensional vector bundle. Let $H$ be the structure group of $E$ which may be infinite dimensional. First assume that the fiber of $E$ is an irreducible representation of $H$. We say $E$ is a vector bundle of positive energy, if

1. There exists a fiberwise $S^{1}$-action on $E$, such that under this action $E$ is decomposed into direct sum of finite dimensional vector bundles, $\oplus_{n \in Z} E_{n}$;

2. The character $\chi(\tau)=\sum_{n} \operatorname{dim} E_{n} q^{n+a}$ for some rational number a is a modular form.

This definition is motivated by the definition of positive energy representations of infinite dimensional Lie algebras $[\mathrm{K}]$ and Lie groups [PS]. In general, we also call the direct sum and tensor product of two positive energy bundles of positive energy.

The examples of positive energy bundles include the case that $H=\tilde{L} G$ with $G$ a simple and simply connected Lie group. Each positive energy representation of loop group admits an action of Diff ${ }^{+} S^{1}$ which, in our case extends to fiberwise action on the corresponding infinite dimensional vector bundle on $M$. Therefore in loop group case, one has very rich structures on the infinite 
dimensional vector bundles. On the other hand, the Virasoro equivariant bundles on $L M$ in $[\mathrm{Br}]$, when restricted to $M \subset L M$, gives infinite dimensional vector bundles of positive energy on $M$.

Corresponding to each positive energy vector bundle $E$, we introduce a formal power series with genuine vector bundle coefficients, $\psi(E)=q^{a} \sum_{n} E_{n} q^{n}$ and consider the set $\{\psi(E)\}$ for all $E$ 's. The sum and product in this set are induced by the sum and the product of the corresponding formal power series. In the standard way, we can make it into an abelian group with respect to the sum. Together with product, it becomes a ring which we denote by $F K(M)$. Especially interesting is the subring generated by those elements coming from positive energy vector bundles with loop group as structure group, we denote this subring by $L K(X)$. Obviously the equivalence relation in $F K(X)$ and $L K(X)$ is not the one induced from the $K$-group of $X$.

3.2. Riemann-Roch and Virasoro algebra. Let $X, Y$ be two compact smooth spin manifolds with an embedding $f: Y \rightarrow X$. Assume that $\operatorname{dim} Y=2 l$ and $\operatorname{dim} X=2 k$. Denote by $F H^{*}(X)$ the image of the Chern character

$$
\operatorname{ch}: F K(X) \rightarrow H^{*}(X, R)
$$

where $R$ is the ring

$$
R=\lim _{N \rightarrow \infty} Q\left[\left[q^{-1 / N}, q^{1 / N}\right]\right] .
$$

Let $f_{*}$ and $f^{*}$ denote the push-forward and pull-back map in cohomology respectively.

The following theorem is an analogue of the Atiyah-Hirzebruch RiemannRoch theorem [AH1].

Theorem 3.1. Assume $f^{*} p_{1}(X)=p_{1}(Y)$, then for any element $U$ in $F K(Y)$, there exists an element $f_{!} U$ in $F K(X)$, such that

$$
f_{*}(\hat{\Theta}(Y) \operatorname{ch} U)=\hat{\Theta}(X) \operatorname{ch~} f_{!} U
$$

holds in $F H^{*}(X)$.

We can also consider the subring $L K(X)$ in $F K(X)$ generated by those elements coming from positive energy vector bundles with loop group as structure group. We can replace $F K(X)$ by $L K(X)$ in which case the above theorem still holds. More precisely we have

Theorem 3.2. Assume $f^{*} p_{1}(X)=p_{1}(Y)$, then for any element $U$ in $L K(Y)$, there exists an element $f_{!} U$ in $L K(X)$, such that

$$
f_{*}(\hat{\Theta}(Y) \operatorname{ch} U)=\hat{\Theta}(X) \operatorname{ch} f_{!} U
$$

holds in $L H^{*}(X)$. 
Here $L H^{*}(X)$ denotes the image of the Chern character of $L K(X)$ in $H^{*}(X, R)$. Note that every element in $L K(X)$ admits a fiberwise action of Diff ${ }^{+} S^{1}$. Especially one can take $X=S^{2 L}$ for a big integer $L$ and $Y$ an $M O<8>$-manifold to get the

Corollary 3.1. Every elliptic genus of $Y$ can be realized as a (virtual) infinite dimensional vector bundle on $S^{2 L}$ on which there exists a fiberwise action of Diff ${ }^{+}{ }^{1}$.

Here virtual means the difference of two bundles and the $D i f f^{+} S^{1}$ acts on each of them. The proof of Theorems 3.1 and 3.2 are basically the same as the proof of the Atiyah-Hirzebruch Riemann-Roch. We use the existence of the level 1 positive energy representations of $\tilde{L} \operatorname{Spin}(2 l), S^{+}, S^{-}$such that the character of $S^{+}-S^{-}$is given by

$$
\chi_{S^{+-} S^{-}}=\prod_{j=1}^{l} \frac{\theta\left(\alpha_{j}, \tau\right)}{\eta(\tau)}
$$

as in $\S 1.2$. For a different proof of Corollary 3.1, see [Ta].

Tensor category may enter this picture in the following way. Take a principal bundle on a manifold $M$ with structure group $\tilde{L} G$ with $G$ a semisimple Lie group. Let $P_{m}(G)$ be the set of highest weight representations of of $\tilde{L} G$ of level $m$. Each element in $P_{m}(G)$ induces one element in $L K(X)$ by the above construction. We denote the set consisting of these elements by $L K_{m}(M, G)$.

There exists an interesting exotic tensor structure on $P_{m}(G)$ which is given by fusion rule $[\mathrm{KL}]$. This naturally induces an exotic tensor structure on $L K_{m}(M, G)$. For complex Grassmanniann, this is in turn isomorphic to the quantum cohomology. According to $[\mathrm{KL}]$, as tensor category $P_{m}(G)$ is isomorphic to the tensor category of representations of the quantum group $U_{q} G$ with $q$ a root of unity. $U_{q} G$ is the deformation of the universal eveloping algebra of $G$. Hopefully the three very interesting subjects, loop groups, quantum groups and tensor category may bring some new light to topology.

3.3. Vector bundles and Jacobi forms. This section is motivated by the vertex operator algebra construction of the monstrous moonshine module. We will discuss a construction from Jacobi forms and relate it to elliptic cohomology.

Let $R(G)$ be the Grothendieck ring of representations of a Lie group $G$. Let $q=e^{2 \pi i \tau}$ with $\tau$ in the upper half plane be a parameter. Let $\left\{V_{n}\right\}$ be a family of elements in $R(G)$ and $\chi\left(V_{n}\right)$ denote their characters.

A Jacobi form of level $m$ and weight $k$ with $l$ variables over $L \rtimes \Gamma$, where $L$ is an integral lattice in the complex plane $C$ preserved by the modular subgroup $\Gamma$ is a holomorphic function $f(t, \tau)$ with $t=\left(t_{1}, \cdots, t_{l}\right)$ on $C^{l} \times H$ such that 
(1) $f\left(\frac{t}{c \tau+b}, \frac{a \tau+b}{c \tau+d}\right)=(c \tau+d)^{k} e^{2 \pi i m c \sum_{j} t_{j}^{2} /(c \tau+d)} f(t, \tau)$,

(2) $f(t+\lambda \tau+\mu, \tau)=e^{-2 \pi i m\left(l \lambda^{2} \tau+2 \lambda \sum_{j} t_{j}\right)} f(t, \tau)$

where $(\lambda, \mu) \in L$ and $g=\left(\begin{array}{ll}a & b \\ c & d\end{array}\right) \in \Gamma$. Here

$$
\frac{t}{c \tau+d}=\left(\frac{t_{1}}{c \tau+d}, \cdots, \frac{t_{l}}{c \tau+d}\right)
$$

and

$$
t+\lambda \tau+\mu=\left(t_{1}+\lambda \tau+\mu, \cdots, t_{l}+\lambda \tau+\mu\right) .
$$

Let $\left(x_{1}, \cdots, x_{l}\right)$ denote the standard root basis of $G$. Consider the formal power series $\sum_{n} V_{n} \cdot q^{n}$.

Definition: A family $\left\{V_{n}\right\}$ in $R(G)$ is called a Jacobian family of level $n$ and weight $k$ over $L \rtimes \Gamma$, if there exists a rational number a, such that

$$
q^{a} \sum_{n} \chi\left(V_{n}\right) q^{n}
$$

is a Jacobi form in $\left(x_{1}, \cdots, x_{l} ; \tau\right)$ of level $n$ and weight $k$ over $L \rtimes \Gamma$.

Let $M$ be a smooth compact manifold. Let $P$ be a principal $G$ bundle on $M$. From each $V_{n}$ we get a vector bundle on $M$ by associating $V_{n}$ to $P$. For convenience we still denote the corresponding bundle by $V_{n}$. This induces an element in the ring of formal power series in $q$ with vector bundle coefficients. Let us denote the Grothendieck ring generated by these induced Jacobian elements over $L \rtimes \Gamma$ for some $L$ by $J K(M)_{\Gamma}$.

The examples of Jacobian elements include the elements induced from loop group representations.

1) Bundles induced from loop group representations. Let $E$ be an irreducible positive energy represenation of $\tilde{L} G$. According to the rotation of the circle, $E$ has decomosition $\oplus_{j} E_{j}$ and there exists a rational number $a$ such that

$$
q^{a} \sum_{j} E_{j} q^{j}
$$

is Jacobian. Its weight and level depend on $G$ and $E$.

Actually the category of Jacobi elements may be bigger than that of loop group representations.

2) For any Jacobi form over $S L_{2}(Z)$, one can get a Jacobian element. Let $J_{k, m}$ be the ring of Jacobi forms over $L \rtimes S L_{2}(Z)$ for some lattice $L$ [EZ]. For any $f(z, \tau) \in J_{k, m}$, under the action of $-I$ in $S L_{2}(Z)$ we have by definition

$$
f(-z, \tau)=(-1)^{k} f(z, \tau)
$$

which implies that 


$$
\begin{aligned}
& k \text { even, } f(z, \tau)=\sum_{n} P_{n}\left(\xi+\xi^{-1}\right) q^{n} \\
& k \text { odd, } f(z, \tau)=\sum_{n}\left(\xi-\xi^{-1}\right) P_{n}\left(\xi+\xi^{-1}\right) q^{n}
\end{aligned}
$$

where $\xi=e^{2 \pi i z}$ and $P_{n}$ is a polynomial.

Let $E$ be a complex line bundle on $M$, then

$$
F(E, \tau)=\sum_{n} P_{n}\left(E+E^{-1}\right) q^{n} \text { or } F(E, \tau)=\sum_{n}\left(E-E^{-1}\right) P_{n}\left(E+E^{-1}\right) q^{n}
$$

is a Jacobian element over $L \rtimes S L_{2}(Z)$. Apply splitting principle, we can get a Jacobian element from any vector bundle on $M$.

3) The following interesting element from the definition of elliptic genus is a Jacobian element over $L \ltimes \Gamma_{0}(2)$ with $L=\mathbf{Z}+\mathbf{Z} \tau$,

$$
\left[\left(\triangle_{V}^{+}-\triangle_{V}^{-}\right) \otimes_{n=1}^{\infty} \Lambda_{-q^{n}} V_{C}\right] \cdot\left[\left(\triangle_{V}^{+}+\triangle_{V}^{-}\right) \otimes_{m=1}^{\infty} \Lambda_{q^{m}} V_{C}\right]^{-1}
$$

where $V_{C}$ is the complexification of $V$ and $\triangle_{V}^{ \pm}$are the spinor bundles of $V$.

3.4. Relation with elliptic cohomology. The Jacobian elements defined in last section can be easily related to elliptic cohomology. We take the modular subgroup $\Gamma=\Gamma_{0}(2)$ and denote the corresponding subring of level 0 elements by $J K(M)_{\Gamma}^{0}$ and denote $J K(M)_{\Gamma} \otimes Z\left[\frac{1}{2}\right]$ simply by $J K(M)$. The reason to invert 2, which will be clear from the following discussions, is to get a homomorphism from the Landweber-Ravenel-Stong elliptic cohomology [La]. Note that on a $M O<8>$ manifold, every Jacobian element has level 0 . Let $M_{*}$ be the ring of modular forms over $\Gamma_{0}(2)$ with Fourier coefficients in $Z\left[\frac{1}{2}\right]$, and $B$ be the Bott periodicity element in $K\left(S^{8}\right)$. First it is easy to see the following

\section{Lemma 3.4.1.}

$$
J K\left(S^{8}\right)=M_{*} \oplus M_{*}[B] .
$$

Define the reduced $J K$-group to be the kernal of the induced map by the inclusion of a point $x_{0}$ in $M$,

$$
i^{*}: J K(M) \rightarrow J K\left(x_{0}\right) .
$$

It is clear that $J K\left(x_{0}\right)=M_{*}$. Let us denote the reduced $J K$-group by $\tilde{J K}(M)$. For a manifold $N \subset M$, one can accordingly define the relative $J K$-group by $J K(M, N)=\tilde{J K}(M / N)$ which is a subring of formal power series in $q$ with coefficents in $K(M, N)$ and with modularity. It can also be described by the standard difference construction in topological $K$-theory.

As in topological $K$-theory, for $n \geq 1$, using suspension, we define

$$
\tilde{J K}^{-n}(M)=\tilde{J K}\left(S^{n} M\right),
$$




$$
\begin{gathered}
J K^{-n}(M, N)=\tilde{J K}\left(S^{n}(M / N)\right), \\
J K^{-n}(M)=J K^{-n}(M, \phi) .
\end{gathered}
$$

Let $\varepsilon=\varepsilon_{1}=1 / 16 \theta_{2}^{4} \theta_{3}^{4}$ be as in $\S 1.4$, we then have the following simple lemma:

Lemma 3.4.2. The multiplication by $B \varepsilon$ induces an isomorphism

$$
J K^{-n}(M, N) \rightarrow J K^{-n-8}(M, N) .
$$

Consider

$$
J K^{\prime \prime}(M, N)=\sum_{n} J K^{-n}(M, N)
$$

Let

$$
J K^{*}(M, N)=J K^{\prime \prime}(M, N) /(1-B \varepsilon) .
$$

It is clear that

$$
J K^{*}(p t)=M_{*}\left[\varepsilon^{-1}\right]=Z\left[\frac{1}{2}, \delta, \varepsilon, \varepsilon^{-1}\right] .
$$

The following obvious theorem establishes the relation of this ring with elliptic cohomology

Theorem 3.3. There is a natural homomorphism $\Phi$ from $\Omega_{S p i n}^{*}(M)\left[\frac{1}{2}\right]$ to $J K^{*}(M)$ which factorizes through elliptic cohomology.

$\Phi$ is constructed in a standard way as in $[\mathrm{CF}]$. Let

$$
\pi: E \rightarrow B \operatorname{Spin}(8 k)
$$

be the universal spin bundle, $P$ be the corresponding principal bundle and $S^{ \pm}$ be the two level 1 positive energy representations as given in $\S 1.2$. Denote by $S=S^{+}+S^{-}$. Let $\psi\left(P, S^{ \pm}\right)$and $\psi(P, S)$ be the corresponding power series constructed from $S^{ \pm}$and respectively $S$ as in $\S 1.2$. Then

$$
\beta=\left(\pi^{*}\left(\psi\left(P, S^{+}\right) \psi(P, S)^{-1}\right), \pi^{*}\left(\psi\left(P, S^{-}\right) \psi(P, S)^{-1}\right) ; \mu\right)
$$

where $\mu$ is the Clifford multiplication by $e$ at $(x, e) \in E$, is an element in $J K^{*}(M \operatorname{Spin}(8 k))$. Note that $\psi(P, S)$ is invertable in $J K^{*}(\operatorname{BSpin}(8 k))$.

Let

$$
f: S^{8 k-n} \wedge M \rightarrow M \operatorname{Spin}(8 k)
$$

be the map inducing an element $[X]_{f}$ in $\Omega_{\text {Spin }}^{*}(M)$, then

$$
\Phi\left([X]_{f}\right)=f^{*} \beta \in J K\left(S^{8 k-n} \wedge M\right) .
$$

This theorem implies the following diagram of natural homomorphisms, 


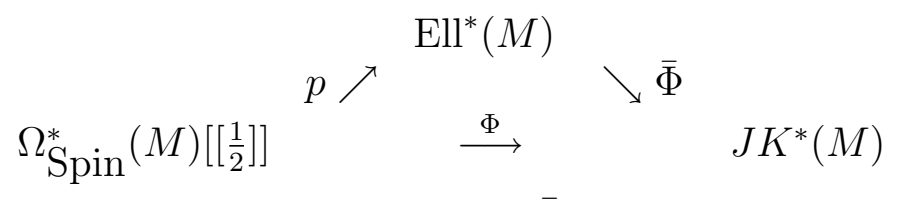

where $p$ is the natural transformation and $\bar{\Phi}$ is the induced map by $\Phi$. This diagram is also compatible with push-forward map [M]. More precisely for $\pi: M \rightarrow p t$, we have the $K$-theory push-forward

$$
\pi_{*}^{K}: J K^{*}(M) \rightarrow M_{*}\left[\varepsilon^{-1}\right],
$$

as well as the elliptic cohomology push-forward

$$
\pi_{*}^{E}: \operatorname{Ell}^{*}(M) \rightarrow M_{*}\left[\varepsilon^{-1}\right] .
$$

For an element $\alpha \in \operatorname{Ell}^{*}(M)$, one has

$$
\pi_{*}^{E} \alpha=\pi_{*}^{K} \bar{\Phi} \alpha
$$

which should be compared with $[\mathrm{M}]$.

It is very easy to see that

$$
\operatorname{Ell}^{*}(M) \otimes Q \backsim J K^{*}(M) \otimes Q .
$$

Actually both of them are isomorphic to $H^{*}\left(M, M_{*}\left[\varepsilon^{-1}\right] \otimes Q\right)$.

Obiviously the $J K^{*}(M)$ is slightly 'bigger' than $\operatorname{Ell}^{*}(M)$. At this point, we do not know how to put an equivalence relation in $J K^{*}(M)$ to make it a cohomology theory. If this can be done properly, then the quotient theory should be very close to a vector bundle description of $\operatorname{Ell}(M)$.

\section{REFERENCES}

[A] Atiyah, M. F.: Collected Works. Oxford Science Publication 1989

[A1] Atiyah, M. F.: Classical Groups and Classical Differential Operators. In [A1], Vol. 4, 341-386

[AB] Atiyah, M. F. and Bott, R.: The Lefschetz Fixed Point Theorems for Elliptic Complexes I, II. in [A] Volume 3 91-170

$[\mathrm{AH}]$ Atiyah, M. F. and Hirzebruch, F.: Spin Manifolds and Group Actions. In [A] Vol. 3 417-429

[AH1] Atiyah, M. F. and Hirzebruch, F.: Riemann-Roch Theorems for Differentiable Manifolds. Bull. Amer. Math. Soc. 65 (1959), 276-281

[AS] Atiyah, M., Singer, I.: The Index of the Dirac Operator. In [A], Vol3 239-300

[BH] Borel, A., Hirzebruch, F.: Characteristic Classes and Homogeneous Spaces I. Amer. J. Math. 80 (1958) 458-538

[BT] Bott, R. and Taubes, C.: On the Rigidity Theorems of Witten. J. of AMS. No.2 (1989) 137-186

[Br] Brylinski, J-L.: Representations of Loop Groups, Dirac Operators on Loop Spaces and Modular Forms. Top. Vol. 29 No. 4 (1990) 461-480 
[Co] Copson, E. T.: Theory of Functions of a Complex Variable. Oxford Univ. Press 1935

[D] Dessai, A.: $S^{3}$-action and the Witten Genus. (Preprint 1993)

[EZ] Eichler, M. and Zagier, D.: The Theorey of Jacobi Forms. Birkhauser 1985

[GL] Gong, D., Liu, K.: On the Rigidity of Higher Elliptic Genera. (Preprint 1994)

[H] Hirzebruch, F.: A Riemann-Roch Theorem for Differentiable Manifolds. Sem. Bourbaki, No. 177 (1959)

[H1] Hirzebruch, F.: Mannigfaltigkeiten und Modulformen. In Jahresber. Deutsch Math.-Verein. Jubilaumstagung, Teubner, Stuttgart, 1990, 20-38.

[HBJ] Hirzebruch, F., Berger, T., Jung, R.: Manifolds and Modular Forms. Vieweg 1991

[K] Kac, V.: Infinite Dimensional Lie Groups. Cambridge Press (1991)

[KL] Kazhdan, D., Luztig, G.: Affine Lie Algebras and Quantum Groups. Int. Math. Res. Notices 21-29 (1991)

[L] Lang, S.: Differential Manifolds. Addison Wesley 1972

[La] Landweber, P. S.: Elliptic Curves and Modular Forms in Algebraic Topology. Lecture Notes in Math. 1326

[LS] Landweber, P., Stong, R.: Circle Actions on Spin Manifolds and Characteristic Numbers. Topology 27 (1988) p 145-161

[Liu] Liu, K.: On Modular Invariance and Rigidity Theorems. J. of Diff. Geom. 1994, Vol. 4

[Liu0] Liu, K.: On Theta-functions and Elliptic Genera. Toplogy, to appear

[Liu1] Liu, K.: Modular Invariance and Characteristic Numbers. Commun. in Math. Physics, to appear

[Liu2] Liu, K.: On $S L_{2}(Z)$ and Topology. Math. Res. Letter Vol.1 No.1 53-64

[LZ] Liu, K., Zhang, W.: Elliptic Genus and $\eta$-invariant. Int. Math. Res. Notice, No. 8 (1994) 319-327.

[M] Miller, H.: The Elliptic Character and the Witten Genus. Contemprory Math. Vol. 96 (1989) 281-289

[O] Ochanine, S.: Sur les Genres Multiplicatifs Definis par Integrales Elliptiques. Topology 26 (1987) 143-151

[S] Segal, G.: Elliptic Cohomology. Seminaire Bourbaki 40eannee, 1987-88 No.695

[Ta] Tamanoi, H.: Tamanoi, H.: Elliptic Genera and Vertex Operator Super Algebras. (Preprint 1994).

[T] Taubes, C.: $S^{1}$-Actions and Elliptic Genera. Comm. in Math. Physics. Vol. 122 No. 3 (1989) 455-526

[We] Weil, A.: Elliptic Functions According to Eisenstein and Kronecker. Springer 1976

[W] Witten, E.: The Index of the Dirac Operator in Loop Space. In [La] 161-186

Department of Mathematics

MIT

Cambridge, MA 02139 